\title{
Composition of matter in the heliosphere
}

\author{
Peter Bochsler ${ }^{1,2}$ \\ ${ }^{1}$ Physikalisches Institut, University of Bern, \\ Sidlerstrasse 5, CH-3012, Bern, Switzerland \\ ${ }^{2}$ Space Science Center, EOS, University of New Hampshire, Durham NH, USA \\ email: bochsler@soho.unibe.ch
}

\begin{abstract}
The Sun is by far the largest reservoir of matter in the solar system and contains more than $99 \%$ of the mass of the solar system. Theories on the formation of the solar system maintain that the gravitational collapse is very efficient and that typically not more than one tenth from the solar nebula is lost during the formation process. Consequently, the Sun can be considered as a representative sample of interstellar matter taken from a well mixed reservoir 4.6 Gy ago, at about $8 \mathrm{kpc}$ from the galactic center. At the same time, the Sun is also a faithful witness of the composition of matter at the beginning of the evolution of the solar system and the formation of planets, asteroids, and comets. Knowledge on the solar composition and a fair account of the related uncertainties is relevant for many fields in astrophysics, planetary sciences, cosmo- and geochemistry. Apart from the basic interest in the chemical evolution of the galaxy and the solar system, compositional studies have also led to many applications in space research, i.e., it has helped to distinguish between different components of diffuse heliospheric matter. The elemental, isotopic, and charge state composition of heliospheric particles (solar wind, interstellar neutrals, pickup ions) has been used for a multitude of applications, such as tracing the source material, constraining parameters for models of the acceleration processes, and of the transport through the interplanetary medium. It is important to realize, that the two mainstream applications, as outlined above - geochemistry and cosmochemistry on one side, and tracing of heliospheric processes on the other side - are not independent of each other. Understanding the physical processes, e.g., of the fractionation of the solar wind, is crucial for the interpretation of compositional data; on the other hand, reliable information on the source composition is the basis for putting constraints on models of the solar wind fractionation.
\end{abstract}

Keywords. Sun:abundances, solar wind, solar system: general, ISM:abundances

\section{Introduction}

Diffuse matter in the heliosphere exhibits a wide diversity of compositional features. Solar matter is considered to be the most representative sample with respect to elemental and isotopic composition of the local galactic interstellar medium (ISM) as it existed 4.6 Gy ago at about $8 \mathrm{kpc}$ from the galactic center. The formation of the Sun can be visualized as a crossroads, at which the galactic nucleosynthetic evolution takes its end and the geochemical evolution of matter in the solar system with all its phenotypes (comets, asteroids, planets, planetary atmospheres etc.) takes its origin. Of course, solar matter has not survived in its pristine chemical state as it prevailed in the form of complex molecules, ices, and dust. In the solar core, where hydrogen has been converted to helium, nuclear reactions have also modified the proportions among other light elements and isotopes. In the outer convective zone (OCZ) some light elements and isotopes such as $\mathrm{D},{ }^{3} \mathrm{He},{ }^{6} \mathrm{Li}$, and ${ }^{7} \mathrm{Li}$ have either been destroyed or - at least - been modified in their abundances. Furthermore, the composition of the OCZ has also been modified to some extent by secular gravitational settling. Next to the Sun, comets are believed to contain the most pristine material of the solar system. Presumably, some of it is still in its 
original chemical form as it was imported as dust and ices from the interstellar medium. However, comets do not contain the volatile inventory of the ISM. Whereas the terrestrial planets have accreted most of their material (including some volatiles) in solids, the most abundant elements hydrogen and helium have been accreted onto the Sun and the giant planets in gaseous form. Elemental and isotopic fractionation during the formation of the Sun must have been negligible as the formation process is thought to be very efficient; i.e., only of the order of $10 \%$ of the mass has been lost and returned to the ISM during the formation of the solar disk. Hence, to a good approximation the notion of "birth of the solar system", i.e. a short event compared to its lifetime is correct: After a short instant of detachment from the ISM, the solar system has remained a closed system.

Certain types of carbonaceous meteorites contain almost all elements in solar proportions (Anders \& Grevesse 1989). The isotopic composition of the non-volatile elements is remarkably similar within this type of meteorites, and it is also comparable with the terrestrial isotopic composition. This indicates a fairly complete homogenization among materials within the inner solar system; an observation which is relevant for inferences on the isotopic composition of these elements also of the Sun - inaccessible to direct measurement. Apart from such inferences it is the solar wind, which provides the most straightforward information on the isotopic composition of solar matter. However, as we will discuss in the following, information on isotopic abundances is biased through fractionation processes during solar wind feeding and acceleration. Experimentally, the isotopic fractionation of the solar wind has been confined by in-situ experiments on moderately refractory elements, which have more than one abundant isotope, and which show little or no variation in meteorites and terrestrial materials, e.g., Mg, Si and Fe (Bochsler et al. 1996, Kallenbach et al. 1998, Kucharek et al. 1998, Ipavich et al. 2001).

The purpose of this review is to summarize the current knowledge on the composition of different types of heliospheric matter, and to show how compositional features can be used to trace their sources.

\section{Sun and solar wind}

The Sun continuously emits charged particles into the surrounding heliosphere. The overwhelming part (95\%) of the ions are protons, the rest consists of alpha particles and heavier species - very roughly in proportions similar to solar composition. Figure 1 shows - as an example - the energy distribution of the solar oxygen flux at 1 AU. The solar wind contributes the by far largest fraction of solar particles. In the energy range above typically $10 \mathrm{keV} /$ nucleon the distribution is populated by the so-called suprathermal particles and by solar energetic particles (SEP's). Suprathermal particles are generated by various processes in the corona and in the interplanetary space, i.e., by shock acceleration at corotating interaction regions (CIRs), or interplanetary shocks, created by the propagation of coronal mass ejections (CME's). Solar particles with higher energies are also generated at reconnection sites of merging solar magnetic loops, by the release of magnetic energy in magnetohydrodynamic instabilities in the solar atmosphere, where so-called "impulsive" events produce energetic particles in abundances strongly deviating from normal coronal composition.

\subsection{Solar wind abundances and solar abundances}

\subsubsection{Observations}

As stated previously, a good agreement has generally been found between meteoritic and solar photospheric abundances. Meteorites contain the refractory elements and isotopes approximately in solar proportions, but they lack the volatile complement. 


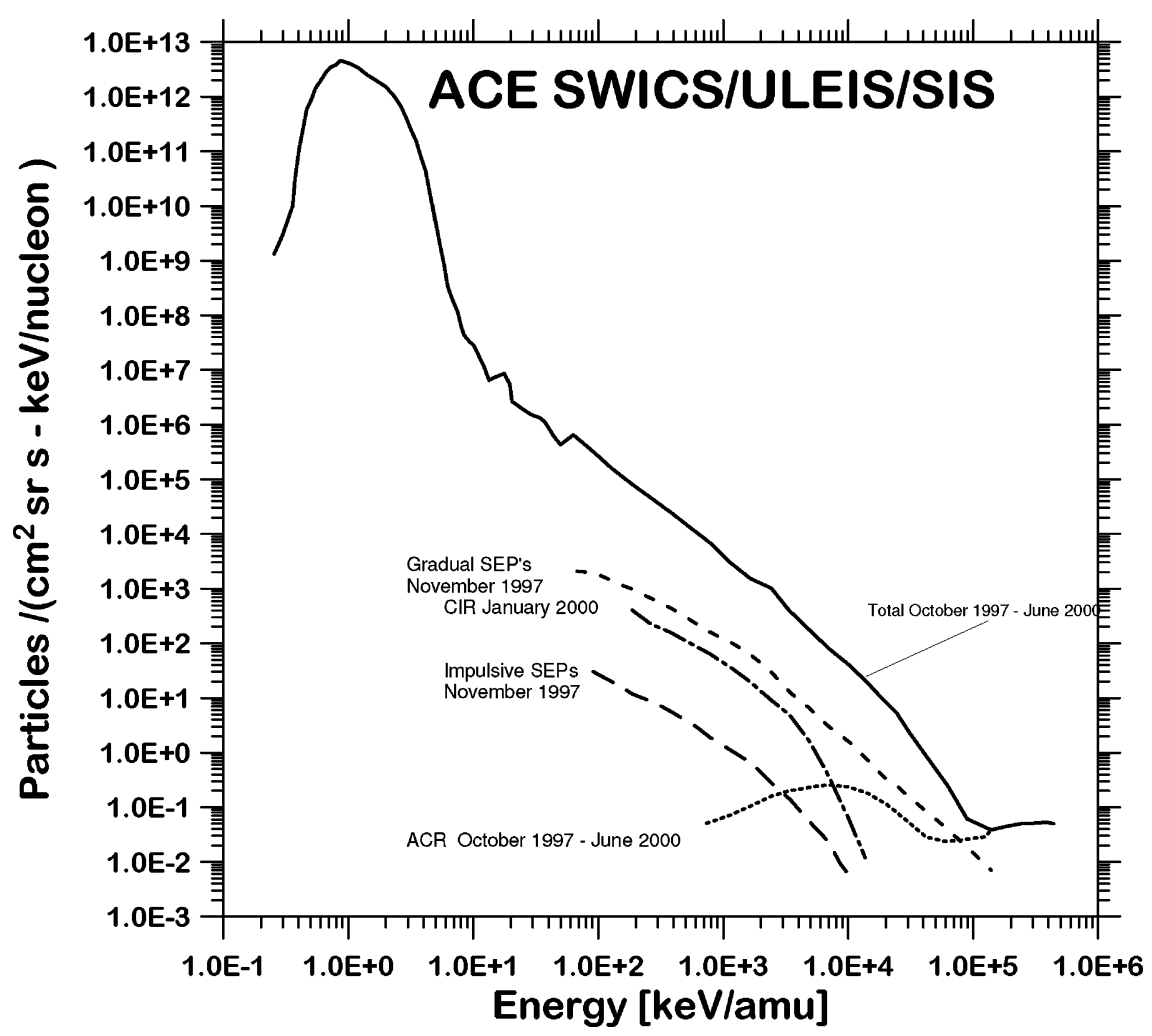

Figure 1. Typical flux of solar oxygen particles as observed with the Advanced Composition Explorer (ACE) from October 1997 through June 2000 at 1 AU. Adapted from Mewaldt et al. (2007).

Therefore, comparisons between the photosphere and meteorites rest essentially on the normalization of a few refractory or moderately refractory key elements such as Si and $\mathrm{Mg}, \mathrm{Fe}$ for which there is on one hand no concern for losses from meteorites (and their parent bodies), and - on the other hand - the photospheric abundances have been determined with fairly high reliability. However, in either case the abundance of these key elements in relation to the most abundant element, hydrogen, is a difficult and sometimes controversial subject. In the case of meteorites - hydrogen is mostly contained in volatile compounds and depleted in all types of meteorites by orders of magnitude - even in the most pristine classes. On the other hand, absolute photospheric spectroscopic abundances rest not only on complex calculations in atomic physics but they also depend strongly on assumptions on parameters of model atmospheres. These models have been steadily improved and the agreement between determinations with the intensity and linewidth of different optical lines has become better (cf. Asplund et al. 2005). Noble gases (He, $\mathrm{Ne}$, and Ar) have high first ionization potentials and, correspondingly, high excitation potentials, and the determination of their abundances by spectroscopic means poses special difficulties. In many compilations of solar system abundances for volatiles one refers therefore to solar wind abundances or to abundances of solar energetic particles. The noble gases in the solar wind have been determined with the Apollo foil experiments (Geiss et al. 1972) and, more recently, with the Genesis sample return mission (Burnett et al. 2003, Grimberg et al. 2006). Laboratory measurements of Apollo- and Genesissamples largely agree with each other and have certainly better precisions than long-time 
averages of in-situ measurements (Bochsler et al. 1986, von Steiger et al. 2000). To infer solar abundances from solar wind noble gases (and also from SEP's) is somewhat more problematic because one needs information on the fractionation mechanisms between the solar atmosphere and the corona. The most notorious and difficult case is the case of helium, which is depleted roughly by a factor 2 in the solar wind compared to the solar atmosphere. This factor is well known, because from helioseismology the helium abundance in the Sun and in the OCZ is known with very high precision and reliability (e.g., Gough 2007). What is less well established is the mechanism, which causes the depletion of helium in the corona and in the solar wind. If this were well understood, it would be possible to infer how much this mechanism affects oxygen, neon, and other elements, which have been well determined in the solar wind, and it would be possible to create a line of evidence for the abundance of heavy elements in the Sun, which is independent of spectroscopic determinations. In fact such an independent line of evidence is badly needed, since the newly determined low abundances of Asplund et al. (2005) have created a problem for helioseismological models. These abundances do not reproduce the observed opacity of solar material. Bahcall et al. (2005) and Antia \& Basu (2006) have made an attempt to fix this abundance problem by raising somewhat arbitrarily the solar neon abundance by a factor of 3 , independent of all other elements. Young (2005) and Bochsler et al. (2006) and Bochsler (2007b) have independently criticized this approach on grounds of optical observations of EUV lines in flares and of solar wind determinations, respectively.

Table 1 is an updated compilation of elemental abundances in the solar wind. For a detailed discussion, see Bochsler (2007a), where also a compilation of isotopic abundances is given.

Table 1. Elemental abundances in the solar wind

\begin{tabular}{|c|c|c|c|}
\hline Element & Interstream & Coronal Hole & References \\
\hline $\mathrm{He}$ & $90 \pm 30$ & $75 \pm 20$ & {$[1,2,3]$} \\
\hline $\mathrm{C}$ & $0.68 \pm 0.07$ & $0.68 \pm 0.07$ & $3]$ \\
\hline $\mathrm{N}$ & $0.078 \pm 0.005$ & $0.114 \pm 0.021$ & {$[3,4]$} \\
\hline $\mathrm{O}$ & $\equiv 1$ & $\equiv 1$ & \\
\hline $\mathrm{Ne}$ & \multicolumn{2}{|c|}{$0.14 \pm 0.03$} & {$[5,6,7,8]$} \\
\hline $\mathrm{Na}$ & $0.0090 \pm 0.0015$ & $0.0051 \pm 0.0014$ & {$[9]$} \\
\hline $\mathrm{Mg}$ & $0.147 \pm 0.050$ & $0.106 \pm 0.050$ & 3 \\
\hline $\mathrm{Al}$ & $0.0119 \pm 0.003$ & $0.0081 \pm 0.0004$ & {$[10]$} \\
\hline $\mathrm{Si}$ & $0.140 \pm 0.050$ & $0.101 \pm 0.040$ & {$[3,11]$} \\
\hline $\mathrm{P}$ & $0.0014 \pm 0.0004$ & & {$[12]$} \\
\hline $\mathrm{S}$ & \multicolumn{2}{|c|}{$0.050 \pm 0.015$} & {$[3,13]$} \\
\hline $\mathrm{Ar}$ & $0.0031 \pm 0.0008$ & $0.0031 \pm 0.0004$ & {$[6,7,14,15]$} \\
\hline $\mathrm{Ca}$ & $0.0081 \pm 0.0015$ & $0.0053 \pm 0.0010$ & {$[16,17,18]$} \\
\hline $\mathrm{Cr}$ & $0.0020 \pm 0.0003$ & $0.0015 \pm 0.0003$ & {$[19]$} \\
\hline $\mathrm{Fe}$ & $0.122 \pm 0.050$ & $0.088 \pm 0.050$ & {$[3,20,21,22]$} \\
\hline $\mathrm{Ni}$ & $0.0065 \pm 0.0025$ & & {$[23]$} \\
\hline
\end{tabular}

References: [1] Bochsler (1984), [2] Bochsler et al. (1986), [3] von Steiger et al. (2000), [4] Gloeckler et al. (1986), [5] Geiss et al. (1972), [6] Geiss et al. (2004), [7] Grimberg et al. (2008), [8] Heber, [9] Ipavich et al. (1999), [10] Bochsler et al. (2000), [11] Bochsler (1989), [12] Giammanco et al. (2008), [13] Giammanco et al. (2007), [14] Cerutti (1974), [15] Weygand et al. (2001), [16] Kern et al. (1997), [17] Kern (1999), [18] Wurz et al. (2003), [19] Paquette et al. (2001), [20] Schmid et al. (1988), [21] Aellig et al. (1999a), [22] Aellig et al. (1999b), [23] Karrer et al. (2007). 


\subsubsection{Theoretical considerations}

How well do the solar wind and the corona reproduce abundances of the OCZ? Understanding the fractionation processes between corona and the solar atmosphere is a necessary ingredient to answer this question. Two main classes of processes have to be considered: The first one is the so-called FIP-Process (FIP = First-Ionization-Potential) (or FIT-First-Ionization-Time), according to which elements can be depleted in the corona depending on their ionization potential. Various models of this mechanism have been proposed, most models contain too many free parameters to make safe predictions and, furthermore, observational data are still of insufficient quality, to eliminate some competing models conclusively. Nevertheless, attempts to establish the importance of this process have been made, by investigating correlations among elements which are expected to be sensitive or not so sensitive to the process. One example is the $\mathrm{He} / \mathrm{H}$ vs $\mathrm{O} / \mathrm{H}$ ratio: $\mathrm{H} / \mathrm{O}$ should be rather insensitive to the FIP-effect, because both elements have the same first ionization potentials and, more importantly, the two elements are expected to be well coupled by resonant charge exchange. Bochsler (2007b) argued that the large variability of $\mathrm{H} / \mathrm{O}$, strongly correlated with $\mathrm{He} / \mathrm{H}$, speaks against a strong influence of the FIP effect not only on $\mathrm{H} / \mathrm{O}$ but also on the $\mathrm{He} / \mathrm{H}$ ratio.

As an alternative, especially in the context of the large $\mathrm{He} / \mathrm{H}$ variability in the solar wind, collisional effects have regularly been considered as the mechanism responsible for this phenomenon. Geiss et al. (1970) have discussed the role of Coulomb friction for accelerating heavy species in the solar wind. Noci \& Porri (1983) later pointed out that the non-negligible abundance of helium is important for the momentum balance, and Bürgi \& Geiss (1986) elaborated their solar wind models adding $\mathrm{He}^{++}$to $\mathrm{H}^{+}$as major particles and found that only the inclusion of helium yields realistic models of the lowspeed solar wind, with the characteristic temperature maxima observed in charge state distributions of minor species. Clearly, Coulomb friction is an important agent in carrying heavy species in the low-speed wind. As a consequence, some isotopic fractionation of low-speed wind compared to photospheric abundances must be considered. From the models of Bodmer \& Bochsler (2000) one can infer that the strongest effect - of the order of a few tens of percents - has to be expected for the case of the helium isotopes. For medium mass range elements, such as $\mathrm{Mg}$, Si, etc., the effect is of the order of a few percent per mass unit - in the sense of a somewhat elevated abundance of the lighter isotopes - but below the limit for an unambiguous detection by currently operating insitu experiments. The effect seems, however, detectable with Genesis (Grimberg et al. 2008).

At the end of this section also the issue of the variability of the solar wind composition should be briefly addressed. The Genesis mission has confirmed the picture of the Apollo foil experiments: The targets exposed in different regimes: low-speed wind, high-speed wind, and CMEs, show small but detectable variations. Although the distinction of these regimes by the onboard computer of Genesis has not been ideal, the velocity histograms recorded with these exposures look fairly different from each other, and from the in-situ experience one would expect a wider diversity of compositions, especially for elemental ratios with helium involved. For the most reliable in-situ data Figure 2 shows that the helium abundance variability is limited within a range of about a factor of 5 in $99 \%$ of the cases. Now, helium is the most likely candidate for strong and variable fractionation since it is special in two respects: It has the highest ionization potential, and $\mathrm{He}^{++}$has the least favorable Coulomb-drag factor of all elements. In contrast, it is difficult to conceive how the Sun could fractionate two mass-neighbors with very similar ionization properties such as $\mathrm{Al}$ and $\mathrm{Mg}$, or $\mathrm{Al}$ and $\mathrm{Si}$ efficiently from each other by a factor of 


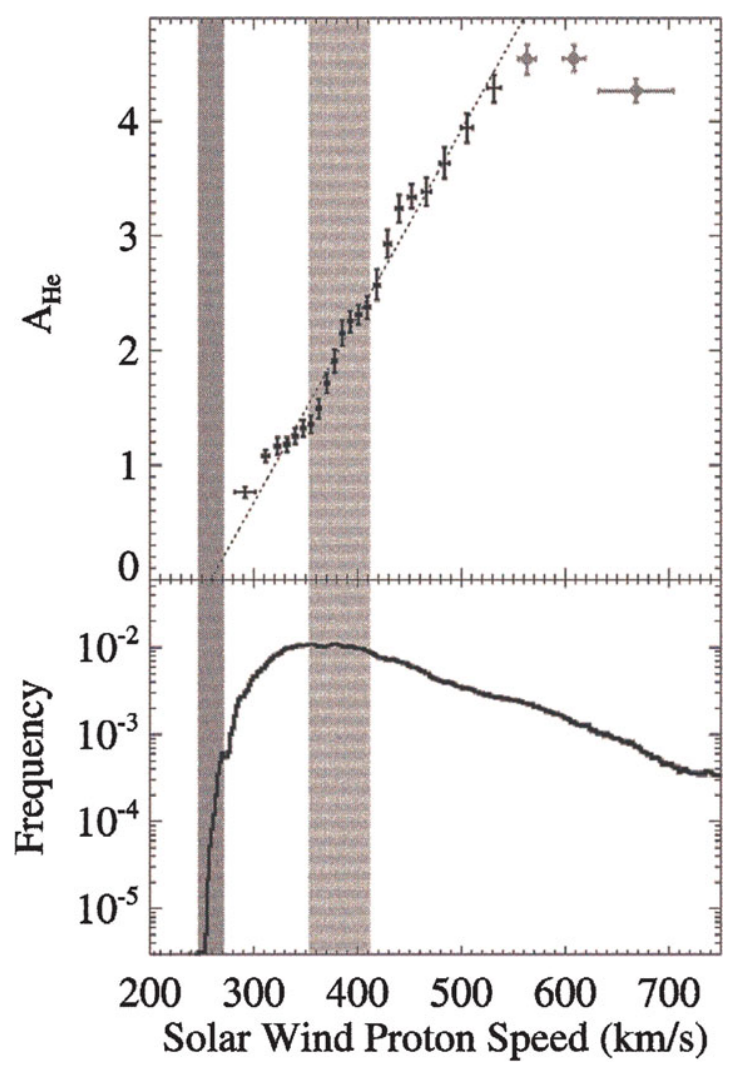

Figure 2. Figure from Kasper et al. (2007) (their Figure 4). The lower panel shows a histogram of speeds registered during the entire Wind mission. The heliomagnetic latitudinal dependence of speeds has been removed. The upper panel shows the corresponding helium abundances as observed with the Wind spacecraft. The expansion factor of the magnetic field low in the corona determines the proton speed and the coupling of protons with helium (and minor ions), thus regulating the helium flux at $1 \mathrm{AU}$.

two or more - even on short time scales, as is sometimes claimed on the basis of in-situ data.

\section{Non-solar heliospheric particles}

\subsection{Interstellar matter in the heliosphere}

A substantial amount of suprathermal and energetic particles in the heliosphere (see Figure 1) originates from so-called pick-up ions from interstellar matter. These particles are particularly apt for acceleration in shock regions as they are produced with velocity distributions substantially wider than the solar wind. Neutral atoms with high ionization potentials can penetrate, unimpeded by the interplanetary magnetic field, deep into the solar system. Occasionally, as they approach the Sun, they are ionized by solar EUV radiation or by charge exchange with solar wind ions. Upon ionization they begin a cycloidal motion about the ambient magnetic field. As the field moves outwards, pickup ions are swept back to the fringes of the heliosphere. Particularly, pickup ions which are pre-accelerated in the innermost heliosphere, can become energized up to several 
$\mathrm{MeVs} / \mathrm{amu}$ at the heliospheric termination shock, and reenter the heliosphere as the socalled anomalous component of cosmic rays (ACR's, lowermost dotted line in Figure 1).

\subsection{Discovery of the "Outer Source"}

From the historical perspective it is interesting to note that in several cases compositional features and charge state distributions of ions have helped to identify the origin of heliospheric particles. For instance, it has been possible to identify the source of ACR's by the fact that it contained an elemental mix, which differs substantially from what is known as "cosmic" composition. Oxygen and nitrogen were significantly overabundant over $\mathrm{C}$ which has a relatively low ionization potential. Fisk et al. (1974) proposed that these particles originated from weakly charged pick-up ions from the interstellar medium, which move out to the heliospheric bow-shock, where they are re-accelerated and brought back as ACR's into the inner solar system. Later, also elements with low ionization potentials have been identified in ACR's (Reames 1999 and Cummings et al. 2002), and it became clear that these elements could not have been imported from the ISM. This led Schwadron et al. (2002) to propose an "Outer Source" of pickup ions for ACRs. Sputtering of interplanetary dust with solar wind ions would release elements with low first ionization potentials and (and consequently, low volatility) out of dust grains such as Si, $\mathrm{Mg}$, and produce the observed enhancement of these elements in the ACR-population (Reames 1999 and Cummings et al. 2002).

Similarity of compositional features with known populations may sometimes lead to erroneous interpretations as has recently been discovered by Grimberg et al. (2006). An enigmatic component of neon - called "SEP-Neon" - had been found in lunar fines (Wieler et al. 1986). Its origin was ascribed to solar energetic particles because it was more deeply implanted in lunar grains than solar wind, and it had an isotopic composition, similar to SEP's. An intriguing problem of this interpretation was its overabundance over several orders of magnitude in relation to the observed fluence of solar wind. Mewaldt et al. (2001) had pointed out the possibility that implantation effects could modify the solar wind isotopic composition to the degree that it appeared in a stepwise release process or an etching procedure similar to solar energetic particles. Grimberg et al. (2006) and Grimberg et al. (2008) now found that this dubious SEP-component was present also in the Genesis target materials despite the fact that the fluence of SEP's during the Genesis exposures was much too small. They confirmed with SRIM-simulations (Ziegler 2004) that indeed - upon implantation into a solid target - the distribution of solar wind particles could be modified in such a way as to produce the observed depth profiles.

\subsection{The "Inner Source"}

Inner Source pickup ions are thought to originate from the interaction of solar wind ions with dust in the inner solar system (Geiss et al. 1996). An unsolved puzzle is the overabundance of neon in inner-source pickup ions reported by Gloeckler et al. (2000) and by Allegrini et al. (2005). Compared to solar wind abundances neon is overrepresented over the neighboring elements C,N,O, and Mg (cf. Figure 4). This is somewhat surprising since one would expect that an interaction of solar wind ions with dust would rather lead to the opposite, i.e. an overrepresentation of dust-constituting elements such as found in the outer source. In general, interplanetary dust seems rather fragile, and furthermore, according to Kehm et al. (2006) far from saturated with noble gases. Consequently, sputtering of solids is expected to produce abundant low-FIP pickup ions. A possible solution to this problem might be the relatively large second ionization rates of $\mathrm{O}, \mathrm{C}$, and Si compared to Ne. Pickup ions from these elements would undergo second ionization 

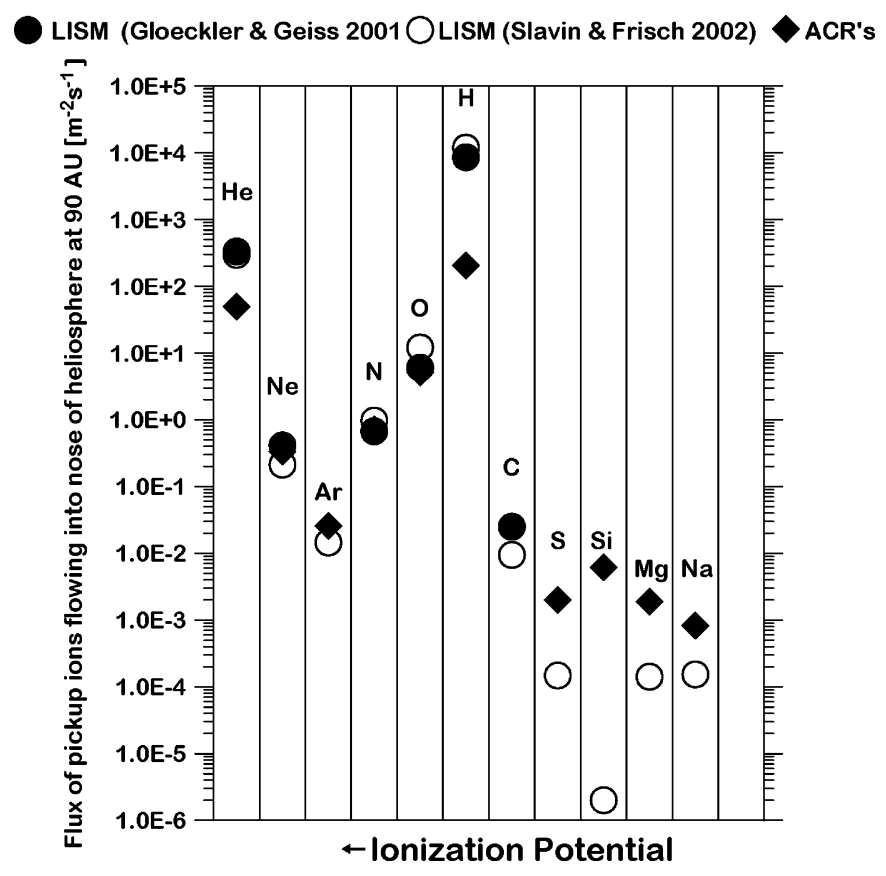

Figure 3. Figure adapted from Cummings et al. (2002) (their Figure 10 and Table 4). The anomalous component apparently consists of two different constituents. Both originate from pickup ions, which are generated in the inner solar system and swept out of the heliosphere with the interplanetary magnetic field. At the termination shock pre-accelerated particles are further energized, and some of them re-enter the heliosphere at energies, typically of the order of a few $\mathrm{MeV} / \mathrm{amu}$, as the so-called anomalous component of cosmic rays (ACR). Using an elaborated set of ionization rates, the elemental abundances of the LISM derived from Gloeckler \& Geiss (2001) (full circles) and from Slavin \& Frisch (2002) (empty circles), Cummings et al. (2002) succeed to model the abundance pattern of high-FIP elements of ACRs (full diamonds) up to oxygen quite accurately. However, ACR's are overabundant for predominantly dust forming, low-FIP elements ( $\mathrm{S}, \mathrm{Si}, \mathrm{Mg}, \mathrm{Na}$ ), thus requiring another origin of these particles, the so-called "Outer Source" of pickup ions as explained by Schwadron et al. (2002).

and become difficult to be detected when they reach the site of observation. This, however, would require a large production or advection of small dust grains in the inner corona (i.e., at heliocentric distances $\leqslant 10 \mathrm{R}_{\odot}$ ) where photoionization rates are high and/or the electron density is still sufficiently high for collisional ionization (Bochsler et al. 2007).

\subsection{Energetic neutrals (ENA)}

With the advent of the technique of surface ionization as a diagnostic tool in space physics, the study of ENA's has become increasingly important. The advantage of neutrals for heliospheric diagnostics is their ability to travel unimpeded through electric and magnetic fields, hence, they can carry information about the source processes, in which they are produced, over large distances across the heliosphere to a remote observer. The backside of the coin is that the detection and analysis of ENA's becomes difficult. For these reasons, compositional analysis of ENAs has still been limited. For a detailed review see Wurz (2000).

Within the inner corona collisional ionization and recombination maintain an equilibrium charge state distribution for a given element. Further out, where the time between collisions with electrons becomes larger than the typical solar wind expansion time, the charge state "freezes" and the static equilibrium can no longer be maintained 


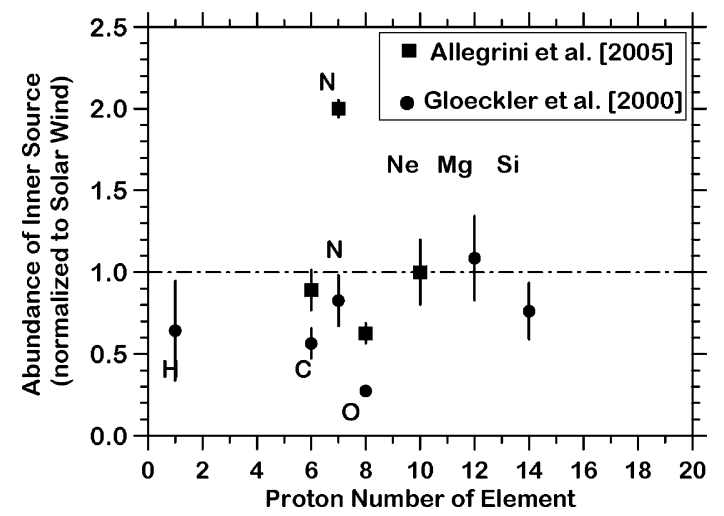

Figure 4. Abundances of Inner Source particles normalized to solar wind abundances and to neon. Full circles are for data from Ulysses/SWICS, published by Gloeckler et al. (2000), full squares from the compilation of Allegrini et al. (2005) of Ulysses/SWICS data.

(e.g., Owocki et al. 1983). Under normal coronal conditions a fraction of the order of $10^{-6}$ of solar wind particles are expected to flow as neutrals. Assuming a proton flux of $5 \cdot 10^{12} \mathrm{~m}^{-2} \mathrm{~s}^{-1}$, one estimates a neutral hydrogen flux of typically $10^{7} \mathrm{~m}^{-2} \mathrm{~s}^{-1}$. Collier et al. (2001) and Collier et al. (2003) reported a fraction of $10^{-3}$ to $10^{-5}$ of neutrals in the solar wind flux at $1 \mathrm{AU}$.

One efficient way of converting solar wind ions into neutrals is backscattering from dust grains. Assuming a typical backscattering and neutralization efficiency of $P_{n}=10 \%$, and assuming that all backscattered particles are converted into neutrals, one can estimate the probability of a solar wind ion to be backscattered and neutralized, not considering for the moment - the possibility of re-ionization by charge exchange and photoionization from solar EUV. We assume a heliospheric radial density distribution of dust grains according to $\mathrm{n}(\mathrm{r}) \propto \mathrm{r}^{\alpha}$. The differential probability of a solar wind ion to hit a dust grain between $\mathrm{r}$ and $\mathrm{r}+\mathrm{dr}$ is then

$$
d p=d r \cdot n(r) \sigma=n(R) \sigma \cdot R\left(\frac{r}{R}\right)^{-\alpha},
$$

where $\mathrm{R}$ is $1 \mathrm{AU}$ and $\mathrm{n}(\mathrm{R}) \sigma$ denotes the integrated cross section density of dust grains at 1 AU. We assume this value to be $5 \cdot 10^{-19} \mathrm{~m}^{2} / \mathrm{m}^{3}$ (Leinert \& Grün 1990). Integrated over the range, from where a neutral particle has a chance to survive as neutral at $r_{0}=0.1$ $\mathrm{AU}$ to the observer at $1 \mathrm{AU}$, one finds a probability

$$
p=P_{n} \frac{n(R) \sigma R}{\alpha-1}\left(\left(\frac{r_{0}}{R}\right)^{1-\alpha}-1\right) .
$$

With a typical value of $\alpha=1.3$ (Leinert \& Grün 1990) one obtains then $\mathrm{p}=3 \cdot 10^{-8}$, which is far below the observed lower limit of Collier et al. (2003) of $10^{-5}$. Again, as in the previous section on the Inner Source, one could seek remedy in increasing the exponent of the density distribution to a probably unrealistic value of $\alpha=4.7$, thereby concentrating the interplanetary dust towards the Sun. Unfortunately, it has not been possible to identify the composition of this neutral solar wind component. At this time the most likely explanation seems solar wind protons, recombined with electrons in the inner corona, although this process seems to provide flux ratios, which are still at least one magnitude under the observed ones. 


\section{Conclusions}

It has been discovered in recent years that, apart from the solar wind and energetic particles of solar origin, the inner heliosphere is hosting a rich diversity of particles of different sources. Many of them have not yet been identified and explained unambiguously. In all cases the determination of their masses and charge states, together with the investigation of their dynamical properties have provided the essential clues. Heliospheric particles are not just protons and electrons. Understanding their properties and their sources requires also a detailed knowledge of their composition.

\section{Acknowledgements}

The author gratefully acknowledges helpful discussions with Berndt Klecker, Ansgar Grimberg, and Rainer Wieler. This work has been supported by NASA STEREO contract NAS5-00132.

\section{References}

Aellig, M. R., Hefti, S., Grünwaldt, H., et al. 1999a, J. Geophys. Res., 104, 24,769

Aellig, M. R., Holweger, H., Bochsler, P., et al. 1999b, in Solar Wind Nine, ed. S. R. Habbal, R. Esser, J. V. Hollweg, \& P. A. Isenberg (AIP Proceedings 471), 255-258

Allegrini, F., Schwadron, N. A., McComas, D. J., Gloeckler, G., \& Geiss, J. 2005, J. Geophys. Res., 110, A05105, doi:10.1029

Anders, E. \& Grevesse, N. 1989, Geochim. Cosmochim. Acta, 53, 197

Antia, H. M. \& Basu, S. 2006, Astrophys. J., 644, 1292

Asplund, M., Grevesse, N., \& Sauval, A. J. 2005, in ASP Conf. Ser. 336: Cosmic Abundances as Records of Stellar Evolution and Nucleosynthesis, ed. T. G. Barnes \& F. N. Bash, 25

Bahcall, J. N., Basu, S., \& Serenelli, A. M. 2005, Astrophys. J., 631, 1281

Bochsler, P. 1984, Habilitationsschrift, University of Bern

Bochsler, P. 1989, J. Geophys. Res., 94, 2365

Bochsler, P. 2007a, Astron. Astrophys. Rev., 14, 1

Bochsler, P. 2007b, Astron. Astrophys., 471, 315

Bochsler, P., Auchère, F., \& Skoug, R. M. 2006, in Proc. SOHO 17 Conference, Taormina (ESA SP 617)

Bochsler, P., Geiss, J., \& Kunz, S. 1986, Solar Phys., 103, 177

Bochsler, P., Gonin, M., Sheldon, R. B., et al. 1996, in Solar Wind Eight. Proceedings of the Eighth International Solar Wind Conference, ed. D. Winterhalter, J. T. Gosling, S. R. Habbal, W. S. Kurth, \& M. Neugebauer, Vol. 382 (Woodbury, N.Y., USA: American Institute of Physics), 199-202

Bochsler, P., Ipavich, F. M., Paquette, J. A., Weygand, J. M., \& Wurz, P. 2000, J. Geophys. Res., 105, 12659

Bochsler, P., Möbius, E., \& Wimmer-Schweingruber, R. F. 2007, in Proc. Second Solar Orbiter Conference, Athens (ESA SP-641)

Bodmer, R. \& Bochsler, P. 2000, J. Geophys. Res., 105, 47

Bürgi, A. \& Geiss, J. 1986, Solar Phys., 103, 347

Burnett, D. S., Barraclough, B. L., Bennett, R., et al. 2003, Space Sci. Rev., 105, 509

Cerutti, H. 1974, PhD Thesis, University of Bern, Switzerland

Collier, M. R., Moore, T. E., Ogilvie, K., et al. 2003, in Proceedings of the Tenth International Solar Wind Conference, ed. M. Velli, R. Bruno, \& F. Malara (Melville, New York: AIP Proceedings 679), 790-793

Collier, M. R., Moore, T. E., Ogilvie, K. W., et al. 2001, J. Geophys. Res., 106, 24,893

Cummings, A. C., Stone, E. C., \& Steenberg, C. D. 2002, Astrophys. J., 578, 194

Fisk, L. A., Kozlovsky, B., \& Ramaty, R. 1974, Astrophys. J., 190, L35

Geiss, J., Bühler, F., Cerutti, H., Eberhardt, P., \& Filleux, C. 1972, Apollo 16 Prel. Sci. Rep. NASA Special Publication, 315, 14.1 
Geiss, J., Bühler, F., Cerutti, H., et al. 2004, Space Sci. Rev., 110, 307

Geiss, J., Gloeckler, G., \& von Steiger, R. 1996, Space Sci. Rev., 78, 43

Geiss, J., Hirt, P., \& Leutwyler, H. 1970, Solar Phys., 12, 458

Giammanco, C., Bochsler, P., Karrer, R., et al. 2007, Space Sci. Rev., 130, 329

Giammanco, C., Wurz, P., \& Karrer, R. 2008, Astrophys. J., 681, 1703

Gloeckler, G., Fisk, L. A., Geiss, J., Schwadron, N. A., \& Zurbuchen, T. H. 2000, J. Geophys. Res., 105, 7459

Gloeckler, G. \& Geiss, J. 2001, in Acceleration and transport of energetic particles observed in the Heliosphere, ed. R. A. Mewaldt, J. R. Jokipii, M. A. Lee, E. Moebius, \& T. H. Zurbuchen, Vol. 528 (Woodbury, N.Y.: American Institute of Physics), 281-

Gloeckler, G., Ipavich, F. M., Hamilton, D. C., et al. 1986, Geophys. Res. Lett., 13, 793

Gough, D. O. 2007, Astron. Nachr./AN, 328, 273

Grimberg, A., Baur, H., Bochsler, P., et al. 2006, Science, 314, 1133

Grimberg, A., Baur, H., Bühler, F., Bochsler, P., \& Wieler, R. 2008, Geochim. Cosmochim. Acta, 72,626

Ipavich, F. M., Bochsler, P., Lasley, S. E., Paquette, J. E., \& Wurz, P. 1999, EOS Trans. AGU, 80,256

Ipavich, F. M., Paquette, J. A., Bochsler, P., Lasley, S. E., \& Wurz, P. 2001, in Solar and Galactic Composition, ed. R. F. Wimmer-Schweingruber, Vol. CP-598 (Melville, N.Y.: AIP Conf. Proceedings), 121-126

Kallenbach, R., Ipavich, F. M., Kucharek, H., et al. 1998, Space Sci. Rev., 85, 357

Karrer, R., Bochsler, P., Giammanco, C., et al. 2007, Space Sci. Rev., 130, 317

Kasper, J. C., Stevens, M. L., Lazarus, A. J., Steinberg, J. T., \& Ogilvie, K. W. 2007, Astrophys. J., 660,901

Kehm, K., Flynn, G. J., \& Hohenberg, C. M. 2006, Meteoritics and Planetary Sciences, 41, 1199

Kern, O. 1999, PhD Thesis, University of Bern, Switzerland

Kern, O., Wimmer-Schweingruber, R. F., Bochsler, P., Gloeckler, G., \& Hamilton, D. C. 1997, in Proceedings of the 31st ESLAB Symp., 'Correlated Phenomena at the Sun, in the Heliosphere and in Geospace', Workshop on Plasma Dynamics and Diagnostics in the Solar Transition Region and Corona (ESA SP-415), 345-348

Kucharek, H., Ipavich, F. M., Kallenbach, R., et al. 1998, 103, 26'805

Leinert, C. \& Grün, E. 1990, In: Physics of the Inner Heliosphere, ed. R. Schwenn \& E. Marsch (Berlin Heidelberg: Springer-Verlag), 207-275

Mewaldt, R. A., Ogliore, R. C., Gloeckler, G., \& Mason, G. M. 2001, in Solar and Galactic Composition, ed. R. F. Wimmer-Schweingruber, Vol. CP-598 (Melville, N.Y.: AIP Conf. Proceedings), 393-398

Mewaldt, R. A., Cohen, C. M., Mason, G. M., Haggerty, D. K., \& Desai, M. I. 2007, Space Sci. Rev., 130, 323

Noci, G. \& Porri, A. 1983, IAGA, Hamburg, paper 4L.04 presented at the 18th General Assembly Meeting

Owocki, S. P., Holzer, T. E., \& Hundhausen, A. J. 1983, Astrophys. J., 275, 354

Paquette, J. A., Ipavich, F. M., Lasley, S. E., Bochsler, P., \& Wurz, P. 2001, in Solar and Galactic Composition, ed. R. F. Wimmer-Schweingruber, Vol. CP-598 (Melville, N.Y.: AIP Conf. Proceedings), $95-100$

Reames, D. V. 1999, Astrophys. J., 518, 473

Schmid, J., Bochsler, P., \& Geiss, J. 1988, Astrophys. J., 329, 956

Schwadron, N. A., Combi, M., Huebner, W., \& McComas, D. J. 2002, Geophys. Res. Lett., 29, doi:10.1029/2002GL015829

Slavin, J. D. \& Frisch, P. C. 2002, Astrophys. J., 565, 364

von Steiger, R., Schwadron, N. A., Hefti, S., et al. 2000, J. Geophys. Res., 105, 27,217

Weygand, J. M., Ipavich, F. M., Wurz, P., Paquette, J. A., \& Bochsler, P. 2001, in Solar and Galactic Composition, ed. R. F. Wimmer-Schweingruber, Vol. CP-598 (Melville, N.Y.: AIP Conf. Proceedings), 101-106

Wieler, R., Baur, H., \& Signer, P. 1986, Geochmim. Cosmochim. Acta, 50, 1997

Wurz, P. 2000, in The outer heliospere: Beyond the planets, ed. K. Scherer, H. Fichtner, \& E. Marsch (Katlenburg-Lindau, Germany: Copernicus Gesellschaft e.V.), 251-288 
Wurz, P., Bochsler, P., Paquette, J. A., \& Ipavich, F. M. 2003, Astrophys. J., 583, 489

Young, P. R. 2005, Astron. Astrophys., 444, L45

Ziegler, J. 2004, Nucl. Instr. and Methods in Phys. Res. B, 219-220, 1027

\section{Discussion}

HowARD: Do the models for the "inner source" include the "dust free region", in which the dust particles are completely vaporized/ionized?

BOCHSLER: The models we developed assume a simplified radial stationary dust density distribution which ends at 5 solar radii. As long as dust is vaporized and ionized in this region, it produces inner source pick-up ions. Dust which is swept away by the interplanetary magnetic field or by radiation pressure, is less efficient in producing pickup ions. Hence, it is important to know which of these processes is more important in eliminating dust. The argument in favor of locating the solar-wind-dust interaction very close to the Sun is the apparent overabundance of neon among the singly ionized pick-up ions. Neon has a bigger chance of leaving the corona as a singly ionized species than dust forming elements $\mathrm{CNO}, \mathrm{Mg}$, Si which tend to become multiply ionized and are much more difficult to detect at 1 AU than singly ionized species.

FISK: You may wish to note that MESSENGER is providing new observations which appear contradictory to our earlier understanding. 